\title{
Virtual Reality Technology in Rural Landscape Design and Value Analysis
}

\author{
Su Tingting ${ }^{1, a, *}$, Shu Xiaoxia ${ }^{1}$, Zhang Dongqian ${ }^{2}$ \\ ${ }^{1}$ Chengdu Agricultural College, Chengdu, Sichuan, 611130, China \\ ${ }^{2}$ Beijing Tsinghua Tongheng Urban Planning \& Design Institute Co.,Ltd. Beijing, 100085, China \\ a email: stt312312@sina.com \\ *corresponding author
}

Keywords: Virtual Reality Technology, Rural Landscape Design, Application and Value

\begin{abstract}
With the development of modern technology in our country, the landscape design of rural gardens will change dramatically with the change of times. Virtual reality technology is a new technology which can fully reflect the development of modern high technology. Its principle is to use computer technology to simulate a virtual three-dimensional space world, which makes it more convenient for human-computer interaction operation. Based on the introduction of virtual reality technology and its application in rural landscape design, this paper emphatically introduces its practical application value in rural landscape design by analyzing the advantages and disadvantages of virtual reality technology.
\end{abstract}

\section{Introduction}

With the rapid development of our country's economy and society, the speed of our country's rural construction is accelerating, at the same time, the degree of environmental greening in the countryside is paid more and more attention to, and the rural areas pay more and more attention to the coordinated development of the environment, but there are still many problems to be solved in the current planning and development of the rural landscape. Therefore, it is very important to strengthen the rural landscape design in the study of rural construction and development. Virtual reality technology is a kind of three-dimensional simulation space which can construct a similar to the real world through computer simulation technology, and can provide some sensory simulation about vision, touch and hearing, which greatly facilitates human-computer interaction operation, and more comprehensively and efficiently embodies the designer's design idea and design intention. Many advantages of virtual reality technology make it become the development trend of landscape design and application field of rural landscape quickly [1].

\section{Development of Virtual Reality Technology}

\subsection{The Concept of Virtual Reality Technology}

Virtual reality technology, also called Lingjing technology, is a new high-tech practical technology developed in the 20th century. Virtual reality technology integrates computer, simulation technology and electronic information. Its basic way of realization is to give people a real sense of touch, vision and hearing through the virtual three-dimensional environment simulated by computer, which can make the audience have a real sense of environmental immersion. Virtual reality technology uses real data in real life to generate certain electronic signals by using computerrelated technology, and combines them with various output devices, thus transforming them into phenomena that can be truly felt by users. These phenomena can include a lot, can be real objects in the real world, and can also be some kind of human invisible material. Virtual reality technology presents these substances through computer 3D model [2].

Virtual reality technology involves a wide range of subjects and complex types of systems, which are determined by its research object and application requirements. After a long period of 
development and improvement, virtual reality technology has developed into a fairly mature hightech technology, and has been recognized by more and more users and developers. Through the use of virtual reality technology, users can get the most real feeling in the virtual reality world, now the virtual reality technology can achieve its authenticity and the real world can not distinguish the true and false degree, so that users can have the real feeling of being on the spot. In addition, virtual reality technology can perceive the perceptual functions that human beings have, such as touch, taste, vision and so on, which strengthens its authenticity. Finally, virtual reality technology has a very powerful simulation system, so that the real sense of human-computer interaction, so that users in the process of use, can operate at will, and can get the most real feedback of the environment.

\subsection{Characteristics of the Virtual Reality Technology System}

Virtual reality technology has many unique characteristics, making it a rare application technology.

Immersion

Immersion is one of the most important features of VR technology. This means that users can become and feel part of the virtual environment created by the computer simulation system in the process of using the technology. The immersion of the virtual reality technology depends on the user's own perception system. In the process of using the technology, the user can feel the sensory stimulation from the virtual world, thus creating a certain resonance phenomenon in the thinking, resulting in the psychological immersion of the user, in the senses like immersion in the real world [3].

\section{Interaction}

The interaction of virtual reality technology refers to the user's maneuverability of the objects in the simulated environment and the natural degree of feedback from the environment. When the user enters the virtual world of computer simulation, the corresponding technology can make the interaction between the user and the surrounding environment through sensors.

Multi-perception

The multi-sensory meaning of virtual reality technology implies that computer technology has many ways of perception, such as human touch, hearing and so on. The ideal virtual reality technology should have all the perceptual functions that human beings have. However, this can not yet be achieved because of the technical constraints in certain areas of expertise.

Conceptual

The concept of virtual reality technology is also called imagination, in which users can interact with the surrounding virtual objects in a certain way, thus achieving the goal of broadening the range of cognition. Virtual reality technology can create an environment that does not exist in the real world, that is, can be based on human creative imagination, dynamic display or sensor to give back to the scene that can not happen in the real world, which also greatly inspired human creativity.[4].

\section{Autonomy}

The autonomy of virtual reality technology refers to the degree to which objects in the virtual environment of computer simulation can act according to the corresponding physical laws. It is precisely because of these characteristics of VR technology that it is becoming more and more popular with users.

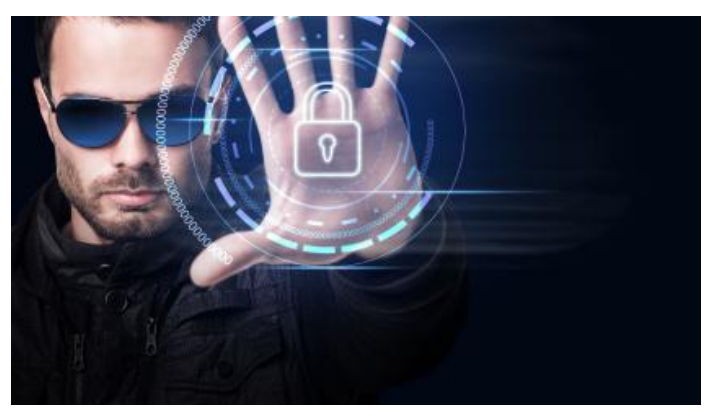

Figure 1 Virtual reality technology 


\section{The Value and Characteristics of Virtual Reality Technology in Rural Landscape Design}

\subsection{The Value of Virtual Reality Technology in Rural Landscape Design}

In the process of rural landscape design, it is necessary to combine the overall planning of the surrounding environment of the countryside, and fully consider the requirements of the users and the overall aesthetic design, so this is a relatively complex series of projects, including the planning, design, construction and maintenance tasks throughout the construction process, which requires the design builder to make a very clear plan for every step of the project and the environment after completion, and to plan the details and the budget of the actual operation process. And virtual reality technology can build a virtual world similar to the real world through the simulation function of the computer, which greatly increases the work efficiency of the related staff, because the virtual world can constantly modify and reconstruct, thus simplifying a series of complex workflow, and also improving the design accuracy and engineering quality of the whole rural landscape construction project.

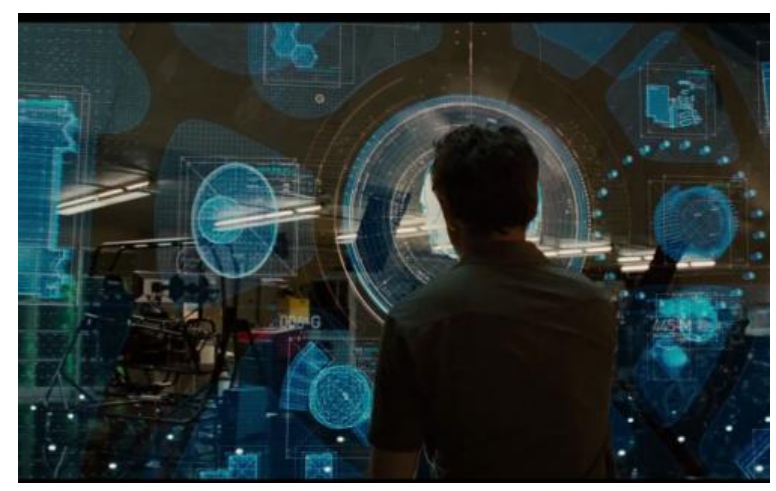

Figure 2 Virtual world

\subsection{Characteristics of Virtual Reality Technology in Rural Landscape Design}

Virtual reality technology can display the landscape space in the rural landscape design, and use the contemporary three-dimensional expression to show every building and environmental details of the whole garden accurately. At the same time, it can also let the designers produce the real landscape space feeling, so as to feel the beauty of the design of the garden from each different route and even the observation angle, and better carry out the landscape design through the experience of the scene. In the design process of the actual landscape, the virtual reality works can be published on the network for the construction unit to watch through the way of VRNL, which is more convenient for the communication between the designer and the construction side, which greatly saves the communication time and reduces the misunderstanding on the communication. In addition, in the whole process of rural landscape design, virtual reality technology can ensure that designers can find and solve the problems in time in the whole design process, because virtual reality technology can enable designers to optimize each space and material details by simulating the real world environment and sensory experience, so as to make corresponding modifications to the less satisfied places, and improve the authenticity and experience of the whole design. Finally, virtual reality technology can also provide designers with dynamic, real-time multiple schemes to compare, assist decision-making, and combine with public participation to provide more ideas for designers' design works and design ideas in rural landscape design, which greatly improves the scientific nature of design and makes the design scheme more humanized and rational.

\section{The Practical Application of Virtual Reality Technology in Rural Landscape Design}

virtual reality technology provides a strong technical support for designers in rural landscape design. with the help of computer assistance, through the establishment of a real rural landscape architecture system simulation system, users can get the experience of immersive experience, so that they can experience the landscape after planning and design more realistically and conveniently. 


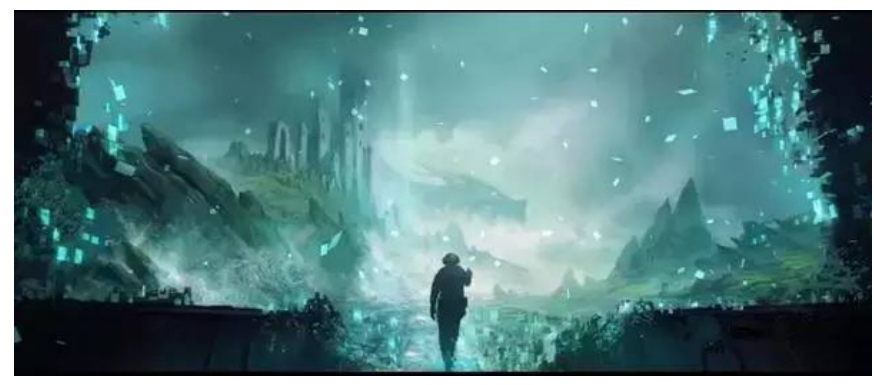

Figure 3 Virtual Park

Feel the space of the landscape in the movement. Through the dynamic and static experience of the simulation space, we can have a whole and continuous feeling and impression on it. Through the real-time interaction ability of the virtual reality technology, the designer can experience the spatial autonomy, at the same time, obtain the real experience of the whole space's vision and hearing, and obtain the real space, the object details from different observation angles and different routes. Visual changes, sensory changes in heat and cold, foot touch and so on have played a great role in the user's space experience. At the same time, in the process of landscape design, all kinds of design elements can be dynamically edited in real time, some parts of the color and structure changes, but also can be real-time environmental layout and environmental greening.

\section{Conclusion}

Although there are still many problems to be solved in the use of virtual reality technology in rural landscape design, with the development of virtual reality technology and the active participation of the vast number of rural landscape designers, the gradual formation of a unified application system will certainly make the rural landscape design more innovative.

\section{References}

[1] Wang Jianning, Wang Tze. virtual reality (VR) in landscape design. Tianjin Agricultural Science, vol. 2303, pp. 103-105, 2017.

[2] Wang Siyuan, Wu Danzi. Virtual reality technology in the course of "landscape architecture design" teaching. Forestry Education in China, vol. 3703, pp. 51-55, 2019.

[3] Wang Yue. How to introduce VR technology in landscape design. Modern Horticulture, vol. 09, pp. 198-199, 2019.

[4] Ding Jingling. virtual reality technology in landscape design. Information and Computer (Theoretical Edition), vol. 3121, pp. 13-16+19, 2019. 